SCIENTIFIC LETTER

\title{
Prognostic implications of sonographic characteristics of carotid plaques in patients with acute coronary syndromes
}

\author{
R Komorovsky, A Desideri, S Coscarelli, L Cortigiani, D Tonello, A Visonà, L Celegon
}

Heart 2005;91:819-820. doi: 10.1136/hrt.2004.042283

W have previously shown that carotid disease is associated with adverse outcomes of patients with acute coronary syndromes (ACS). ${ }^{1}$ The prognostic impact of the sonographic characteristics of carotid plaques, however, remains unclear. The purpose of the present prospective study was to evaluate the relation between ultrasonographic tissue characteristics of carotid plaques and the outcomes of patients with ACS.

\section{METHODS}

The initial study population consisted of 398 consecutive patients admitted to our coronary care unit because of an ACS. Of them, 21 patients had significant valvar disease, six patients had a history of carotid surgery, nine patients had a history of stroke, 17 patients were over 85 years old, three patients refused coronary angiography, and five patients were excluded for logistic reasons. Consequently, 337 patients entered the study. ST elevation acute myocardial infarction (MI) was diagnosed in $102(30 \%)$ patients, non-ST elevation acute MI in 147 (44\%) patients, and unstable angina in 88 $(26 \%)$ patients. Along with the coronary angiography, these patients underwent Doppler ultrasonographic assessment of carotid arteries. Both procedures were performed within 15 days of the acute coronary event. According to the decision of attending physicians, the patients were either managed conservatively ( $\mathrm{n}=123,36 \%$ ), or underwent revascularisation with coronary artery bypass grafting $(n=92,27 \%)$, or percutaneous coronary intervention $(n=122,36 \%)$.

Coronary angiography was performed with the standard femoral or brachial technique. The extent of coronary artery disease was classified as one, two, or three vessel disease according to the number of major coronary arteries with significant $(\geqslant 70 \%)$ stenosis. Significant left main artery stenosis was defined as $\geqslant 50 \%$ lumen narrowing and scored as two vessel disease.

Carotid ultrasound examinations were performed using Sonos 5500 Hewlett Packard equipment with a $7.0 \mathrm{MHz}$ linear array transducer. All examinations were performed by the same sonographer who was blinded to coronary angiography results. Plaques were identified as a focal widening of the vessel wall $>50 \%$ relative to adjacent segments, with protrusion into the lumen. Plaque echogenicity was graded from 1 to 5 according to Gray-Weale classification in the modification of Geroulakos and colleagues. $^{2}$ Plaques of types 1 and 2 were defined as soft (echolucent) plaques, and plaques of types 3 and 4 were defined as hard (echogenic) plaques. Plaques with acoustic shadowing artefact caused by excessive calcification (type 5 plaques) were considered as hard plaques.

Follow up data were collected following a review of the patient's hospital chart, contact with the patient's physician, telephone interview with the patient, and patients' visits to outpatient clinic. Target events were cardiac death and nonfatal MI.

\section{RESULTS}

Carotid plaques were revealed in $144(43 \%)$ of patients. Of them, $45(31 \%)$ patients had soft plaques, and 99 (69\%) patients had hard plaques. The groups were of similar age, had similar values of left ventricular ejection fraction, did not differ significantly with regard to the prevalence of risk factors (hypertension, diabetes mellitus, dyslipidaemia, smoking), one, two, or three vessel coronary artery disease, and treatment strategies. The proportion of medications (statins, $\beta$ blockers, angiotensin converting enzyme inhibitors, aspirin) taken during follow up were similar in both groups. Nineteen (13\%) patients had carotid stenosis $>50 \%$, and nine patients $(6 \%)$ had carotid stenosis $>70 \%$. Bilateral carotid disease was revealed in 116 patients $(79 \%)$.

Follow up was completed for all 337 patients who had enrolled in the study. Median time from enrolment to last contact with patient or target event was 19 months (interquartile range 12-24 months). During follow up, 13 patients $(4 \%)$ experienced cardiac death and 17 patients $(5 \%)$ had a non-fatal MI. Three non-cardiac deaths (one patient from a stroke and two from cancer) and three non-fatal strokes were registered. Among the patients who were free of carotid disease, the rates of major cardiac events were similar to those observed in the whole study population; cardiac death occurred in three $(2 \%)$ of 193 patients, and seven patients (4\%) suffered a non-fatal MI. There was an unfavourable association between the presence of hard carotid plaques and adverse cardiac outcomes; cardiac death or non-fatal MI occurred in 18 of 99 patients (18\%) with hard carotid plaques, compared with two of 45 patients (4\%) with soft carotid plaques $(p=0.02)$. No association was found between intima-media thickness and outcomes.

Treatment stratified Cox multivariate stepwise analysis was applied for prediction of events. Clinical and angiographic data (age, sex, hypertension, diabetes, smoking, hypercholesterolaemia, history of MI, left ventricular ejection fraction, multivessel coronary artery disease) and sonographic characteristics of carotid plaques were considered. Independent predictors of events were smoking (odds ratio (OR) 5.66, p = 0.01), left ventricular ejection fraction (OR $0.03, \mathrm{p}=0.04$ ), hard carotid plaques (OR 1.82, $\mathrm{p}=0.006)$. We compared two multivariate models: model $1\left(\chi^{2}=20.4\right.$, degrees of freedom $(\mathrm{df})=9$, log-likelihood of final solution $=-112.2, \mathrm{p}=0.015)$ containing clinical and angiographic data only and model $2\left(\chi^{2}=28.4, \mathrm{df}=10\right.$, loglikelihood $=-108.2, \mathrm{p}=0.0016$ ) obtained after the addition of sonographic characteristics of carotid plaques to model 1. The incremental value of the latter additional covariant was analysed using $\chi^{2}$ test comparing the loglikelihood of the two models and results were significant $(\mathrm{p}<0.05)$. Kaplan-Meier curves showed a significantly lower event-free survival of patients with hard carotid plaques compared with patients without carotid disease and those with soft carotid plaques (fig 1). 

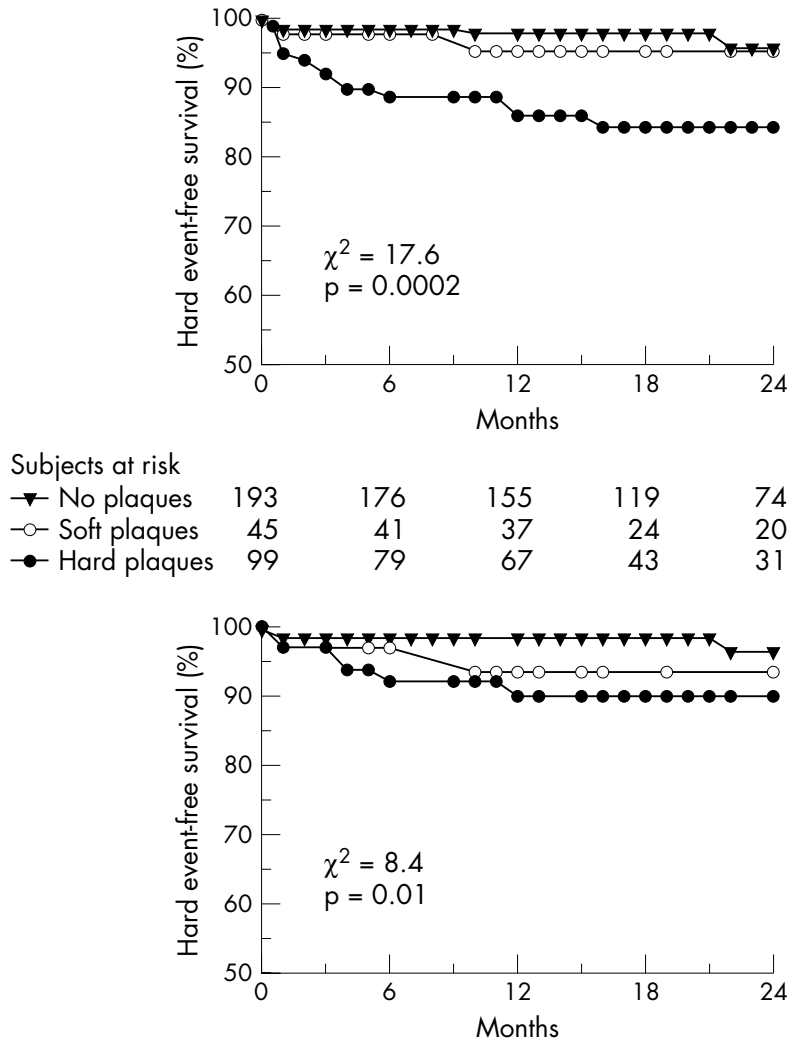

Subjects at risk

$\rightarrow$ No plaques 116

-o- Soft plaques 32

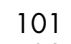

29

53

84

26

65

$\rightarrow$ - Hard plaques 66

45

74

31

Figure 1 Kaplan-Meier estimates of hard event (cardiac death and non-fatal MI)-free survival of the whole study population (upper graph) and of the revascularised patients (lower graph), according to presence or absence of carotid plaques and their sonographic characteristics.

\section{DISCUSSION}

This is the first study evaluating the role of carotid plaque sonographic tissue characterisation in predicting outcomes of patients ACS. Our main finding is that patients with ACS who have hard carotid plaques are characterised by a significantly worse outcome compared with those that have soft plaques or no plaques. Sonographic characteristics of carotid plaques have an incremental value over clinical and angiographic data.
Several hypotheses might explain why hard carotid plaques, as compared with soft carotid plaques, are associated with worse cardiovascular outcomes in patients with ACS. A recent study has shown that hard echogenic plaques are independently associated with increased arterial stiffness in subjects with subclinical carotid atherosclerosis. Increased arterial stiffness, in its turn, has been reported to be a predictor of cardiovascular morbidity and mortality in hypertensive patients. ${ }^{3}$

As yet, the interrelations between amounts of carotid and coronary calcium have not been studied. Calcified carotid plaques might reflect the presence of extensive coronary calcifications. The latter, when assessed by electron beam computer tomography, have been associated to an increased likelihood of cardiac events. ${ }^{4}$

Finally, carotid calcifications may also reflect diffuse coronary disease. The extent of non-obstructive atherosclerotic lesions has been found to be a strong predictor of coronary events, independent of severity and the number of flow limiting coronary lesions. ${ }^{5}$

In conclusion, hard carotid plaques are independently associated with an increased risk of cardiac death or MI in patients with ACS. The results of our study suggest that ultrasound evaluation of carotid arteries may be helpful for risk stratification in ACS patients.

\section{Authors' affiliations}

R Komorovsky, A Desideri, S Coscarelli, L Celegon, Cardiovascular Research Foundation, S Giacomo Hospital, Castelfranco Veneto, Italy D Tonello, A Visonà, Department of Angiology, S Giacomo Hospital L Cortigiani, Department of Cardiology, Campo di Marte Hospital, Lucca, Italy

Correspondence to: Roman Komorovsky, MD, Cardiovascular Research Foundation, Ospedale S Giacomo, 31033 Castelfranco Veneto (TV), Italy; roman_komor@yahoo.com

Accepted 26 August 2004

\section{REFERENCES}

1 Komorovsky R, Desideri A, Coscarelli S, et al. Impact of carotid arterial narrowing on outcomes of patients with acute coronary syndromes. Am J Cardiol 2004;93:1552-5.

2 Geroulakos G, Ramaswami G, Nicolaides A, et al. Characterisation of symptomatic and asymptomatic carotid plaques using high-resolution real time ultrasound. Br J Surg 1993;80:1274-7.

3 Zureik M, Bureau JM, Temmar $M$, et al. Echogenic carotid plaques are associated with aortic arterial stiffness in subjects with subclinical carotid atherosclerosis. Hypertension 2003:41:519-27.

4 Keelan PC, Bielak LF, Ashai K, et al. Long-term prognostic value of coronary calcification detected by electron-beam computed tomography in patients undergoing coronary angiography. Circulation 2001;104:412-7.

5 Bigi R, Cortigiani L, Colombo P, et al. Prognostic and clinical correlates of angiographically diffuse non-obstructive coronary lesions. Heart 2003;89:1-5. 